\title{
Cryptanalysis of RSA-type cryptosystems based on Lucas sequences, Gaussian integers and elliptic curves ${ }^{\star}$
}

\author{
Martin Bunder ${ }^{1}$, Abderrahmane Nitaj ${ }^{2}$, Willy Susilo ${ }^{3}$, and Joseph Tonien ${ }^{3}$ \\ 1 School of Mathematics and Applied Statistics, \\ University of Wollongong, Australia \\ mbunder@uow.edu.au \\ 2 Département de Mathématiques, \\ Université de Caen, France \\ abderrahmane.nitaj@unicaen.fr \\ 3 Centre for Computer and Information Security Research, \\ School of Computing and Information Technology, \\ University of Wollongong, Australia \\ [wsusilo, joseph_tonien] @uow.edu.au
}

\begin{abstract}
In 1995, Kuwakado, Koyama and Tsuruoka presented a new RSA-type scheme based on singular cubic curves $y^{2} \equiv x^{3}+b x^{2}(\bmod N)$ where $N=p q$ is an RSA modulus. Then, in 2002, Elkamchouchi, Elshenawy and Shaban introduced an extension of the RSA scheme to the field of Gaussian integers using a modulus $N=P Q$ where $P$ and $Q$ are Gaussian primes such that $p=|P|$ and $q=|Q|$ are ordinary primes. Later, in 2007, Castagnos proposed a scheme over quadratic field quotients with an RSA modulus $N=p q$ based on Lucas sequences. In the three schemes, the public exponent $e$ is an integer satisfying the key equation $e d-k\left(p^{2}-1\right)\left(q^{2}-1\right)=1$. In this paper, we apply the continued fraction method to launch an attack on the three schemes when the private exponent $d$ is sufficiently small. Our experiments demonstrate that for a 1024-bit modulus, our method works for values of $d$ of up to 520 bits. We also examine the effect of dropping the usual assumption that $p$ and $q$ have the same bit size.
\end{abstract}

Keywords: RSA, elliptic curves, continued fractions.

\footnotetext{
* This paper is the extended version of "A new attack on three variants of the RSA cryptosystem" [2] presented at ACISP 2016
} 


\section{Introduction}

The public key cryptosystem RSA was introduced by Rivest, Shamir and Adleman [11] in 1978. It is the most popular and widely used public-key cryptosystem. The RSA operations system are based on modular arithmetic. Let $p$ and $q$ be two large primes. The product $N=p q$ is called the RSA modulus and the product $\phi(N)=(p-1)(q-1)$ is the Euler totient function. In RSA, the public exponent $e$ and the private exponent $d$ are integers satisfying $e d \equiv 1(\bmod \phi(N))$. A message $m$ is encrypted as $c \equiv m^{e}(\bmod N)$ and decrypted using $m \equiv c^{d}$ $(\bmod N)$.

Since its introduction, the RSA cryptosystem has been generalized in various ways, including extensions to singular elliptic curves and Gaussian integers.

In 1995, Kuwakado, Koyama and Tsuruoka [9] presented a new RSA-type scheme based on singular cubic curves with equation $y^{2} \equiv x^{3}+b x^{2}(\bmod N)$ where $N=p q$ is an RSA modulus and $b \in \mathbb{Z} / N \mathbb{Z}$. The public exponent is an integer $e$ such that $\operatorname{gcd}\left(e,\left(p^{2}-1\right)\left(q^{2}-1\right)\right)=1$ and the decryption exponent is the integer $d \equiv e^{-1}\left(\bmod \left(p^{2}-1\right)\left(q^{2}-1\right)\right)$. From this, we deduce that $e$ and $d$ satisfy a key equation of the form $e d-k\left(p^{2}-1\right)\left(q^{2}-1\right)=1$ where $k$ is a positive integer.

In 2002, Elkamchouchi, Elshenawy and Shaban [6] introduced an extension of RSA to the ring of Gaussian integers. A Gaussian integer is a complex number of the form $a+i b$ where both $a$ and $b$ are integers and $i^{2}=-1$. The set of all Gaussian integers is denoted $\mathbb{Z}[i]$. A Gaussian prime number is a Gaussian integer that cannot be represented as a product of non-unit Gaussian integers. The only unit Gaussian integers are $\pm 1, \pm i$. Let $P=a+i b$ and $Q=a^{\prime}+i b^{\prime}$ be two Gaussian primes. Consider the Gaussian integer $N=P Q$ and the Euler totient function $\phi(N)=(|P|-1)(|Q|-1)=\left(a^{2}+b^{2}-1\right)\left(a^{\prime 2}+b^{\prime 2}-1\right)$. Let $e$ be an integer such that $d \equiv e^{-1}(\bmod \phi(N))$ exists. Then, in the RSA scheme over the domain of Gaussian integers, a message $m \in \mathbb{Z}[i]$ is encrypted using $c \equiv m^{e}$ $(\bmod N)$ and decrypted using $m \equiv c^{d}(\bmod N)$. We note that, in this RSA variant, the key equation is $e d-k(|P|-1)(|Q|-1)=1$ for $N=P Q \in \mathbb{Z}[i]$. In the situation that $N=p q$ is an ordinary RSA modulus, the key equation becomes $e d-k\left(p^{2}-1\right)\left(q^{2}-1\right)=1$, which is the same than in the KuwakadoKoyama-Tsuruoka elliptic curve variant of RSA.

In 2007, Castagnos [4] proposed a probabilistic scheme based on an RSA modulus $N=p q$ and using arithmetical operations in quadratic field quotients. Let $e$ be a integer such that $\operatorname{gcd}\left(e,\left(p^{2}-1\right)\left(q^{2}-1\right)\right)=1$. For any integer $r$, let $V_{e}(r)$ be the $e$ th term of the Lucas sequence defined by $V_{0}(r)=2, V_{1}(r)=r$ and $V_{k+2}=r V_{k+1}(r)-V_{k}(r)$ for $k \geq 0$. In this scheme, a message $m \in \mathbb{Z} / N \mathbb{Z}$ is encrypted using $c \equiv(1+m N) V_{e}(r)\left(\bmod N^{2}\right)$ where $r$ is a random integer with $2 \leq r \leq N-2$. Then some arithmetical properties, one can decrypt $c$ to get the original message $m$. Similarly to the Kuwakado-Koyama-Tsuruoka elliptic curve variant of RSA and RSA with Gaussian integers, Castagnos scheme leads to the key equation $e d-k\left(p^{2}-1\right)\left(q^{2}-1\right)=1$.

The security of the RSA cryptosystem and its variants are based on the difficulty of factoring large integers of the shape $N=p q$. Nevertheless, in some 
cases, the modulus $N$ can be factored by algebraic methods that are not based on factoring algorithms. For example, in 1990, Wiener [12] showed how to break the RSA when the decryption exponent $d$ satisfies $d<\frac{1}{3} N^{0.25}$. Wiener's method is based on solving the key equation $e d-k(p-1)(q-1)=1$ by applying the continued fraction algorithm to the public rational fraction $\frac{e}{N}$. When $d$ is small enough, $\frac{k}{d}$ is one of the convergents of the continued fraction expansion of $\frac{e}{N}$. Later, Boneh and Durfee [1] applied lattice reduction and Coppersmith's technique [5] and extended the bound to $d<N^{0.292}$. Recently, using the convergents of the continued fraction expansion of $\frac{e}{N^{\prime}}$ where $N^{\prime}$ is a number depending on $N$, Bunder and Tonien [3] could break the RSA if $d^{2} e<8 N^{1.5}$.

The complexity of the encryption and decryption algorithms are based on the size of the encryption key $e$ and the size of decryption key $d$, respectively. In a cryptosystem with a limited resource such as a credit card, it is desirable to have a smaller value of $d$. In some scenario, for convenience, $e$ is set to a small constant, such as $e=3$.

In this paper, we consider one of the following scenarios where $N=p q$ is the product of two large primes and the public exponent $e$ satisfies an equation $e d-k\left(p^{2}-1\right)\left(q^{2}-1\right)=1$ with a suitably small secret exponent $d$ :

- an instance of the Kuwakado-Koyama-Tsuruoka cryptosystem [9],

- an instance of the RSA over Gaussian integers [6],

- an instance of Castagnos scheme [4].

Our method is inspired by Bunder and Tonien's technique [3]. We show that in the case when $p$ and $q$ has equal bit length, if $d^{2} e<2 N^{3}-18 N^{2}$ then one can find $p$ and $q$ and then factor the modulus $N$. Our method is based on the continued fraction algorithm as in Bunder and Tonien's attack. Under the condition $d<\sqrt{\frac{2 N^{3}-18 N^{2}}{e}}$, we show that one can find $\frac{k}{d}$ among the convergents of the continued fraction expansion of the public rational number $\frac{e}{N^{2}-\frac{9}{4} N+1}$. In the general case, where $q<p<\mu q$, if $d^{2} e<\frac{\mu N^{3}}{(\mu-1)^{2}}$ then we can find $\frac{k}{d}$ among the convergents of the continued fraction expansion of $\frac{e}{N^{2}+1-\frac{(\mu+1)^{2}}{2 \mu} N}$.

The paper is organized as follows. In Section 2, we present the KuwakadoKoyama-Tsuruoka RSA-type scheme, the RSA scheme over Gaussian integers and the Castagnos scheme. In Section 3, we review some facts and lemmas used in our attack. In Section 4 , we present our new attack for the case $q<p<2 q$ and in Section 5, our attack for the general case $q<p<\mu q$. Experiment results for $\mu=2$ and $\mu=6$ are presented in Section 6 . Note that the contents of Section 5 and Section 6 are new and have not been included in our conference paper [2].

\section{Preliminaries}

In this section, we present the three variants of the RSA cryptosystem for which our attack works, namely the Kuwakado-Koyama-Tsuruoka RSA-type scheme, the RSA scheme over Gaussian integers and the Castagnos scheme. 


\subsection{The Kuwakado-Koyama-Tsuruoka RSA-type scheme}

The Kuwakado-Koyama-Tsuruoka RSA-type scheme is based on the use of an RSA modulus $N=p q$ as the modulus of a singular elliptic curve. Let $\mathbb{Z}_{N}=$ $\mathbb{Z} / N \mathbb{Z}$ be the ring of integers modulo $N$ and $\mathbb{F}_{p}$ be the finite field. Let $a$ and $b$ be integers with $\operatorname{gcd}(a b, N)=1$ and $\operatorname{gcd}\left(4 a^{3}+27 b^{2}, N\right)=1$. A singular elliptic curve $E_{N}(a, b)$ over the ring $\mathbb{Z}_{N}$ is the concatenation of a point $\mathcal{O}_{N}$, called the point at infinity, and the set of points $(x, y) \in \mathbb{Z}_{N}^{2}$ satisfying the Weierstrass equation

$$
y^{2}+a x y \equiv x^{3}+b x^{2} \quad(\bmod N) .
$$

If we consider this form modulo $p$, we get an elliptic curve $E_{p}(a, b)$ over $\mathbb{F}_{p}$

$$
E_{p}(a, b): y^{2}+a x y \equiv x^{3}+b x^{2} \quad(\bmod p),
$$

with the point at infinity $\mathcal{O}_{p}$. It is well known that the chord-and-tangent method defines an addition law on singular elliptic curves, as for all elliptic curves on $\mathbb{F}_{p}$. The addition law can be summarized as follows.

- For any point $P \in E_{p}(a, b), P+\mathcal{O}_{p}=\mathcal{O}_{p}+P=P$.

- If $P=(x, y) \in E_{p}(a, b)$, then $-P=(x,-a x-y)$.

- If $P=(x, y)$, then $2 P=P_{3}=\left(x_{3}, y_{3}\right)$ with

$$
\begin{aligned}
& x_{3}=\left(\frac{3 x^{2}+2 b x-a y}{2 a y+a x}\right)^{2}+a\left(\frac{3 x^{2}+2 b x-a y}{2 a y+a x}\right)-b-2 x, \\
& y_{3}=-\left(\frac{3 x^{2}+2 b x-a y}{2 a y+a x}+a\right) x_{3}-\frac{-x^{3}}{2 a y+a x} .
\end{aligned}
$$

- If $P_{1}=\left(x_{1}, y_{1}\right)$ and $P_{2}=\left(x_{2}, y_{2}\right)$ with $P_{1} \neq \pm P_{2}$, then $P_{1}+P_{2}=P_{3}=$ $\left(x_{3}, y_{3}\right)$ with

$$
\begin{aligned}
& x_{3}=\left(\frac{y_{2}-y_{1}}{x_{2}-x_{1}}\right)^{2}+a\left(\frac{y_{2}-y_{1}}{x_{2}-x_{1}}\right)-b-x_{1}-x_{2}, \\
& y_{3}=-\left(\frac{y_{2}-y_{1}}{x_{2}-x_{1}}+a\right) x_{3}-\frac{y_{1} x_{2}-y_{2} x_{1}}{x_{2}-x_{1}} .
\end{aligned}
$$

The addition law can be extended to the elliptic curve $E_{N}(a, b)$ in the same way as the addition in $E_{p}(a, b)$ by replacing computations modulo $p$ by computations modulo $N$. In $E_{N}(a, b)$, a specific problem can occur. Sometimes, the inverse modulo $N$ does not exist. In this case, this could lead to finding a prime factor of $N$, which is unlikely to happen when $p$ and $q$ are large. Note that this is one of the principles of Elliptic Curve Method of factorization [10].

In 1995, Kuwakado, Koyama and Tsuruoka [9] proposed a system based on singular elliptic curves modulo an RSA modulus, which can be summarized as follows. 


\section{Key Generation:}

- Choose two distinct prime numbers $p$ and $q$ of similar bit-length.

- Compute $N=p q$.

- Choose $e$ such that $\operatorname{gcd}\left(e,\left(p^{2}-1\right)\left(q^{2}-1\right)\right)=1$.

- Compute $d=e^{-1}\left(\bmod \left(p^{2}-1\right)\left(q^{2}-1\right)\right)$.

- Keep $p, q, d$ secret and publish $N, e$.

\section{Encryption:}

- Transform the message as $m=\left(m_{x}, m_{y}\right) \in \mathbb{Z}_{N} \times \mathbb{Z}_{N}$.

- Compute $b=\frac{m_{y}^{2}-m_{x}^{3}}{m_{x}^{2}}(\bmod N)$.

- Compute the ciphertext point $\left(c_{x}, c_{y}\right)=e\left(m_{x}, m_{y}\right)$ on the elliptic curve $y^{2}=x^{3}+b x^{2}(\bmod N)$.

\section{Decryption:}

- Compute $b=\frac{c_{y}^{2}-c_{x}^{3}}{c_{x}^{2}}(\bmod N)$.

- Compute the plaintext point $\left(m_{x}, m_{y}\right)=d\left(c_{x}, c_{y}\right)$ on the elliptic curve $y^{2}=x^{3}+b x^{2}(\bmod N)$.

Observe the modular inverse $d=e^{-1}\left(\bmod \left(p^{2}-1\right)\left(q^{2}-1\right)\right)$ can be transformed as a key equation

$$
e d-k\left(p^{2}-1\right)\left(q^{2}-1\right)=1,
$$

which will be the starting equation of our new attack.

\subsection{RSA over the domain of Gaussian integers}

We now focus on how to extend the RSA cryptosystem to the ring of Gaussian integers. We begin by reviewing the main properties of Gaussian integers.

A Gaussian integer is a complex number of the form $a+b i$ where $a, b \in \mathbb{Z}$ and $i^{2}=-1$. The set of all Gaussian integers is the ring $\mathbb{Z}[i]$. Let $\alpha$ and $\beta \neq 0$ be two Gaussian integers. We say that $\beta$ divides $\alpha$ if there exists a Gaussian integer $\gamma$ such that $\alpha=\beta \gamma$. The norm of a Gaussian integer $a+b i$ is $|a+b i|=a^{2}+b^{2}$. A Gaussian prime is a Gaussian integer which is divisible only by a unit. The units in $\mathbb{Z}[i]$ are \pm 1 and $\pm i$ and have norm 1 . As a consequence, if $a^{2}+b^{2}$ is a prime number in $\mathbb{Z}$, then $a+i b$ is a Gaussian prime. Conversely, if $p \in Z$ is an ordinary prime number, then Gaussian integers $p$ and $p i$ are Gaussian primes if and only if $p \equiv 3(\bmod 4)$. The existence of prime factorization in $\mathbb{Z}[i]$ allows us to consider Gaussian integers of the form $N=P Q$ where $P$ and $Q$ are Gaussian primes with large norm. Similarly, the existence of Euclidean division and Euclidean algorithm in $\mathbb{Z}[i]$ allow us to consider arithmetic operations modulo $N$. On the other hand, if $P$ is a Gaussian prime, then $\alpha^{|P|-1} \equiv 1(\bmod P)$ whenever $\alpha \neq \equiv$ $(\bmod P)$. Similarly, if $N=P Q$ is the product of two Gaussian primes, then $\alpha^{(|P|-1)(|Q|-1)} \equiv 1(\bmod N)$ whenever $\alpha \neq \equiv 0(\bmod N)$. In particular, if $N=$ $p q \in \mathbb{Z}$ is the product of two ordinary primes, then $\alpha^{\left(p^{2}-1\right)\left(q^{2}-1\right)} \equiv 1(\bmod N)$ whenever $\alpha \not \equiv 0(\bmod N)$. 
Using the arithmetical operations on the ring $\mathbb{Z}[i]$, Elkamchouchi, Elshenawy and Shaban [6] proposed an extension of the RSA cryptosystem to Gaussian integers. The scheme can be summarized as follows.

\section{Key Generation:}

- Choose two distinct Gaussian primes $P$ and $Q$ of similar norm.

- Compute $N=P Q$.

- Choose $e$ such that $\operatorname{gcd}(e,(|P|-1)(|Q|-1))=1$.

- Determine $\left.d=e^{-1}(\bmod (|P|-1)(|Q|-1))\right)$.

- Keep $P, Q, d$ secret, publish $N, e$.

\section{Encryption:}

- Transform the message as a Gaussian integer $M \in \mathbb{Z}[i]$.

- Compute $C \equiv M^{e}(\bmod N)$.

\section{Decryption:}

- Compute $M \equiv C^{d}(\bmod N)$.

When $N=p q \in \mathbb{Z}$ where $p$ and $q$ are ordinary prime numbers of the form $4 m+3$, the modular inverse of $e$ becomes $d=e^{-1}\left(\bmod \left(p^{2}-1\right)\left(q^{2}-1\right)\right)$ and can be rewritten as

$$
e d-k\left(p^{2}-1\right)\left(q^{2}-1\right)=1 \text {. }
$$

This is the same key equation that comes up in the Kuwakado-Koyama-Tsuruoka RSA-type scheme.

\subsection{Castagnos scheme}

Castagnos scheme [4] was proposed in 2007 and uses an RSA modulus $N=p q$ and a public exponent $e$ such that $\operatorname{gcd}\left(e,\left(p^{2}-1\right)\left(q^{2}-1\right)\right)=1$. The encryption and the decryption algorithms make use of the Lucas series. Let $r$ be an integer. Define $V_{0}(r)=2$ and $V_{1}(r)=r$. For $k \geq 0$, the $k+2$ th term of the Lucas sequence is defined by $V_{k+2}=r V_{k+1}(r)-V_{k}(r)$. The Lucas series can be computed efficiently by the square and multiply algorithm. The Castagnos scheme can be summarized as follows, where $\left(\frac{x}{p}\right)$ is the Jacobi symbol.

\section{Key Generation:}

- Choose two distinct prime numbers $p$ and $q$ of similar bit-length.

- Compute $N=p q$.

- Choose $e$ such that gcd $\left(e,\left(p^{2}-1\right)\left(q^{2}-1\right)\right)=1$.

- Keep $p, q$ secret and publish $N, e$.

\section{Encryption:}

- Transform the message as an integer $m \in \mathbb{Z} / N \mathbb{Z}$.

- Choose a random integer $r \in[2, n-2]$.

- Compute the ciphertext $c \equiv(1+m N) V_{e}(r)\left(\bmod N^{2}\right)$. 


\section{Decryption:}

- Compute $i_{p}=\left(\frac{c^{2}-4}{p}\right)$ and $d\left(p, i_{p}\right) \equiv e^{-1}\left(\bmod p-i_{p}\right)$.

- Compute $i_{q}=\left(\frac{c^{2}-4}{q}\right)$ and $d\left(q, i_{q}\right) \equiv e^{-1}\left(\bmod q-i_{q}\right)$.

- Compute $r_{p} \equiv V_{d\left(p, i_{p}\right)}(\bmod p)$ and $r_{q} \equiv V_{d\left(q, i_{q}\right)}(\bmod q)$.

- Compute $p^{\prime} \equiv p^{-1}(\bmod q)$ and $r=r_{p}+p\left(r_{p}-r_{q}\right) p^{\prime}(\bmod N)$.

- Compute $t_{p} \equiv \frac{c}{V_{e}(r)}\left(\bmod p^{2}\right)$ and $m_{p} \equiv \frac{t_{p}-1}{p} \cdot q^{-1}(\bmod p)$.

- Compute $t_{q} \equiv \frac{c}{V_{e}(r)}\left(\bmod q^{2}\right)$ and $m_{q} \equiv \frac{t_{q}-1}{q} \cdot p^{-1}(\bmod q)$.

- Compute the plaintext $m \equiv m_{p}+p\left(m_{q}-m_{p}\right) p^{\prime}(\bmod N)$.

Despite the inverse $d \equiv e^{-1}\left(\bmod \left(p^{2}-1\right)\left(q^{2}-1\right)\right)$ is not being used directly in the scheme, we use the key equation $e d-k\left(p^{2}-1\right)\left(q^{2}-1\right)=1$ to launch an attack on Castagnos scheme when $d$ is suitably small.

\section{Useful lemmas}

In this section, we review the main properties of the continued fractions and state a useful lemma that will be used in the attack.

A continued fraction is an expression of the form

$$
a_{0}+\frac{1}{a_{1}+\frac{1}{a_{2}+\frac{1}{\ddots}}}
$$

The continued fraction expansion of a number is formed by subtracting away the integer part of it and inverting the remainder and then repeating this process again and again. The coefficients $a_{i}$ of the continued fraction of a number $x$ are constructed as follows:

$$
x_{0}=x, a_{n}=\left[x_{n}\right], x_{n+1}=\frac{1}{x_{n}-a_{n}}
$$

We use the following notation to denote the continued fraction

$$
x=\left[a_{0}, a_{1}, \ldots, a_{n}\right]=a_{0}+\frac{1}{a_{1}+\frac{1}{\ddots+\frac{1}{a_{n}}}}
$$

If $k \leq n$, the continued fraction $\left[a_{0}, a_{1}, \ldots, a_{k}\right]$ is called the $k^{\text {th }}$ convergent of $x$. The following theorem gives us the fundamental recursive formulas to calculate the convergents. 
Theorem 1. [7] The $k^{\text {th }}$ convergent can be determined as

$$
\left[a_{0}, \ldots, a_{k}\right]=\frac{p_{k}}{q_{k}}
$$

where the sequences $\left\{p_{n}\right\}$ and $\left\{q_{n}\right\}$ are specified as follows:

$$
\begin{aligned}
p_{-2}=0, \quad p_{-1}=1, \quad p_{n}=a_{n} p_{n-1}+p_{n-2}, & \forall n \geq 0 \\
q_{-2}=1, \quad q_{-1}=0, \quad q_{n}=a_{n} q_{n-1}+q_{n-2}, & \forall n \geq 0 .
\end{aligned}
$$

Theorem 2. [7] Let $p, q$ be positive integers such that

$$
0<\left|x-\frac{p}{q}\right|<\frac{1}{2 q^{2}}
$$

then $\frac{p}{q}$ is a convergent of the continued fraction of $x$.

Now, we present a useful result that will be used throughout the paper.

Lemma 1. Let $N=p q$ be an $R S A$ modulus with $q<p<2 q$. Let $\phi_{1}=N^{2}+$ $1-\frac{5}{2} N$ and $\phi_{2}=N^{2}+1-2 N$. Then

$$
\phi_{1}<\left(p^{2}-1\right)\left(q^{2}-1\right)<\phi_{2}
$$

Proof. Suppose that $q<p<2 q$. Then $1<\frac{p}{q}<2$, so since the function $f(x)=$ $x+\frac{1}{x}$ is increasing on $[1,+\infty)$, we get $f(1)<f\left(\frac{p}{q}\right)<f(2)$, that is

$$
2<\frac{p}{q}+\frac{q}{p}<\frac{5}{2}
$$

Multiplying by $N$, we get

$$
2 N<p^{2}+q^{2}<\frac{5}{2} N
$$

Since $\left(p^{2}-1\right)\left(q^{2}-1\right)=N^{2}+1-\left(p^{2}+q^{2}\right)$, we get

$$
N^{2}+1-\frac{5}{2} N<\left(p^{2}-1\right)\left(q^{2}-1\right)<N^{2}+1-2 N,
$$

that is $\phi_{1}<\left(p^{2}-1\right)\left(q^{2}-1\right)<\phi_{2}$. This terminates the proof. 


\section{A new attack on RSA variants based on continued fractions}

In this section, we propose a new attack on the Kuwakado-Koyama-Tsuruoka cryptosystem as well as RSA over the Gaussian integer domain and the Castagnos scheme in the situation that the key equation $e d-k\left(p^{2}-1\right)\left(q^{2}-1\right)=1$ is satisfied with a suitably small secret exponent $d$.

Theorem 3. Let $(N, e)$ be a public key in the Kuwakado-Koyama-Tsuruoka cryptosystem or in the RSA cryptosystem with Gaussian integers or in the Castagnos scheme with $N=p q$ and $q<p<2 q$. If $e<\left(p^{2}-1\right)\left(q^{2}-1\right)$ satisfies an equation ed $-k\left(p^{2}-1\right)\left(q^{2}-1\right)=1$ with

$$
d<\sqrt{\frac{2 N^{3}-18 N^{2}}{e}}
$$

then $\frac{k}{d}$ is a convergent of the continued fraction expansion of

$$
\frac{e}{N^{2}-\frac{9}{4} N+1}
$$

and one can factor $N$ in polynomial time.

Proof. Let $\phi_{1}=N^{2}+1-\frac{5}{2} N$ and $\phi_{2}=N^{2}+1-2 N$. Then $N^{\prime}=N^{2}-\frac{9}{4} N+1$ is the midpoint of the interval $\left[\phi_{1}, \phi_{2}\right]$. Since $\left(p^{2}-1\right)\left(q^{2}-1\right) \in\left[\phi_{1}, \phi_{2}\right]$, then

$$
\left|\left(p^{2}-1\right)\left(q^{2}-1\right)-N^{\prime}\right|<\frac{1}{2}\left(\phi_{2}-\phi_{1}\right)=\frac{1}{4} N .
$$

Using the equation $e d-k\left(p^{2}-1\right)\left(q^{2}-1\right)=1$, we get

$$
\begin{aligned}
\left|\frac{e}{N^{\prime}}-\frac{k}{d}\right| & \leq e\left|\frac{1}{N^{\prime}}-\frac{1}{\left(p^{2}-1\right)\left(q^{2}-1\right)}\right|+\left|\frac{e}{\left(p^{2}-1\right)\left(q^{2}-1\right)}-\frac{k}{d}\right| \\
& =e \frac{\left|\left(p^{2}-1\right)\left(q^{2}-1\right)-N^{\prime}\right|}{N^{\prime}\left(p^{2}-1\right)\left(q^{2}-1\right)}+\frac{1}{\left(p^{2}-1\right)\left(q^{2}-1\right) d}
\end{aligned}
$$

Then, using $d=\frac{k\left(p^{2}-1\right)\left(q^{2}-1\right)+1}{e}$ and (3), we get

$$
\left|\frac{e}{N^{\prime}}-\frac{k}{d}\right|<\frac{e N}{4 N^{\prime}\left(p^{2}-1\right)\left(q^{2}-1\right)}+\frac{e}{\left(p^{2}-1\right)\left(q^{2}-1\right)\left(k\left(p^{2}-1\right)\left(q^{2}-1\right)+1\right)} .
$$

Now, using Lemma 1, we get

$$
\left|\frac{e}{N^{\prime}}-\frac{k}{d}\right|<\frac{e N}{4 \phi_{1}^{2}}+\frac{e}{\phi_{1}^{2}}<\frac{e(N+4)}{4\left(\phi_{1}-1\right)^{2}}=\frac{e(N+4)}{4\left(N^{2}-\frac{5}{2} N\right)^{2}} .
$$

A straightforward calculation shows that

$$
\frac{N+4}{4\left(N^{2}-\frac{5}{2} N\right)^{2}}<\frac{1}{4 N^{3}-36 N^{2}}
$$


Combining this with (2), we get

$$
\left|\frac{e}{N^{\prime}}-\frac{k}{d}\right|<\frac{e}{4 N^{3}-36 N^{2}} .
$$

If $d<\sqrt{\frac{2 N^{3}-18 N^{2}}{e}}$, then $\left|\frac{e}{N^{\prime}}-\frac{k}{d}\right|<\frac{1}{2 d^{2}}$ and by Theorem $2, \frac{k}{d}$ is a convergent of the continued fraction expansion of $\frac{e}{N^{\prime}}$. Using $k$ and $d$, we get

$$
\left(p^{2}-1\right)\left(q^{2}-1\right)=\frac{e d-1}{k}
$$

Combining with $N=p q$, we get the values of $p$ and $q$ which leads to the factorization of $N$. Observe that every step in the proof can be done in polynomial time. This terminates the proof.

\section{$5 \quad$ Different bit size primes}

In Section 4, we consider the case where the two primes $p$ and $q$ are of the same bit size, i.e. $q<p<2 q$. In this section, we give a generalised attacked by considering two primes $p$ and $q$ of arbitrary sizes. We will not require $p$ and $p$ to have the same bit size, but instead, we consider the case $q<p<\mu q$ where $\mu$ is a parameter. We show that if

$$
d^{2} e<\frac{\mu N^{3}}{(\mu-1)^{2}}
$$

then the three RSA-variant schemes can be broken.

Theorem 4. Let $(N, e)$ be a public key in the Kuwakado-Koyama-Tsuruoka cryptosystem or in the RSA cryptosystem with Gaussian integers or in the Castagnos scheme with $N=p q$ and $q<p<\mu q$. If $e<\left(p^{2}-1\right)\left(q^{2}-1\right)$ satisfies an equation ed $-k\left(p^{2}-1\right)\left(q^{2}-1\right)=1$ with

$$
d<\frac{N\left(N-\left(\mu+\frac{1}{\mu}\right)\right)}{\sqrt{e\left(\frac{(\mu-1)^{2}}{\mu} N+2\right)}} \approx \frac{\sqrt{\mu} N^{\frac{3}{2}}}{(\mu-1) \sqrt{e}}
$$

then $\frac{k}{d}$ is a convergent of the continued fraction expansion of

$$
\frac{e}{N^{2}+1-\frac{(\mu+1)^{2}}{2 \mu} N}
$$

and one can factor $N$ in polynomial time.

Proof. We have $1<\frac{p}{q}<\mu$, so based on the increasing property of the function $f(x)=x+\frac{1}{x}$ on $[1,+\infty)$, we get $f(1)<f\left(\frac{p}{q}\right)<f(\mu)$, that is

$$
2<\frac{p}{q}+\frac{q}{p}<\mu+\frac{1}{\mu} .
$$


Multiplying by $N$, we get

$$
2 N<p^{2}+q^{2}<\left(\mu+\frac{1}{\mu}\right) N .
$$

Since $\left(p^{2}-1\right)\left(q^{2}-1\right)=N^{2}+1-\left(p^{2}+q^{2}\right)$, we get

$$
N^{2}+1-\left(\mu+\frac{1}{\mu}\right) N<\left(p^{2}-1\right)\left(q^{2}-1\right)<N^{2}+1-2 N .
$$

Let

$$
\begin{aligned}
& \phi_{1}=N^{2}+1-\left(\mu+\frac{1}{\mu}\right) N, \\
& \phi_{2}=N^{2}+1-2 N, \\
& N^{\prime}=N^{2}+1-\frac{(\mu+1)^{2}}{2 \mu} N .
\end{aligned}
$$

Then $N^{\prime}$ is the midpoint of the interval $\left[\phi_{1}, \phi_{2}\right]$. Since $\left(p^{2}-1\right)\left(q^{2}-1\right) \in\left[\phi_{1}, \phi_{2}\right]$, we have

$$
\left|\left(p^{2}-1\right)\left(q^{2}-1\right)-N^{\prime}\right|<\frac{1}{2}\left(\phi_{2}-\phi_{1}\right)=\frac{(\mu-1)^{2}}{2 \mu} N .
$$

Using the equation $e d-k\left(p^{2}-1\right)\left(q^{2}-1\right)=1$, we get

$$
\begin{aligned}
\left|\frac{e}{N^{\prime}}-\frac{k}{d}\right| & \leq e\left|\frac{1}{N^{\prime}}-\frac{1}{\left(p^{2}-1\right)\left(q^{2}-1\right)}\right|+\left|\frac{e}{\left(p^{2}-1\right)\left(q^{2}-1\right)}-\frac{k}{d}\right| \\
& =e \frac{\left|\left(p^{2}-1\right)\left(q^{2}-1\right)-N^{\prime}\right|}{N^{\prime}\left(p^{2}-1\right)\left(q^{2}-1\right)}+\frac{1}{\left(p^{2}-1\right)\left(q^{2}-1\right) d} \\
& =e \frac{\left|\left(p^{2}-1\right)\left(q^{2}-1\right)-N^{\prime}\right|}{N^{\prime}\left(p^{2}-1\right)\left(q^{2}-1\right)}+\frac{e}{\left(p^{2}-1\right)\left(q^{2}-1\right)\left(k\left(p^{2}-1\right)\left(q^{2}-1\right)+1\right)}
\end{aligned}
$$

Then, using (3), we get

$$
\begin{aligned}
\left|\frac{e}{N^{\prime}}-\frac{k}{d}\right| & <\frac{e \frac{(\mu-1)^{2}}{2 \mu} N}{\left(\phi_{1}-1\right)^{2}}+\frac{e}{\left(\phi_{1}-1\right)^{2}} \\
& =\frac{e\left(\frac{(\mu-1)^{2}}{2 \mu} N+1\right)}{\left(\phi_{1}-1\right)^{2}} \\
& =\frac{e\left(\frac{(\mu-1)^{2}}{\mu} N+2\right)}{2 N^{2}\left(N-\left(\mu+\frac{1}{\mu}\right)\right)^{2}} .
\end{aligned}
$$

Combining with the condition

$$
d<\frac{N\left(N-\left(\mu+\frac{1}{\mu}\right)\right)}{\sqrt{e\left(\frac{(\mu-1)^{2}}{\mu} N+2\right)}}
$$


we have

$$
\left|\frac{e}{N^{\prime}}-\frac{k}{d}\right|<\frac{1}{2 d^{2}}
$$

So by Theorem $2, \frac{k}{d}$ is a convergent of the continued fraction expansion of $\frac{e}{N^{\prime}}$, and thus, we can find the factorization of $N$ in polynomial time. This terminates the proof.

\section{Experiment results}

\subsection{Same bit primes}

In connection with Theorem 3, we present an experimental result. We consider two RSA primes $p$ and $q$, both of 512 bits, which give us a 1024-bit modulus $N$. The public exponent $e$ is 2029-bit and the secret exponent $d$ is 520-bit. We can verify that the condition $d<\sqrt{\frac{2 N^{3}-18 N^{2}}{e}}$ is satisfied as required by Theorem 3 .

$$
\begin{aligned}
p= & 10099263457471330007198432945318371291445329854502 \\
& 15792059491972427206683240499403879190564150845276 \\
& 8065924106792289081626662457201616730699111723973185481 \\
q= & 92010524322086390067138686626606399738950237269245 \\
& 68782613825773843108268162152815137070448098390827 \\
& 116142067687814447541784724352584064538977073778553491 \\
N= & 92923852598882409829316208222552906482402932610895 \\
& 27967459304569281602253526177460006245420686015350 \\
& 58200190869644402726093196331924622411826579351302 \\
& 07820537360725528276550397315706429397980805113181 \\
& 16311534625580255681045311678091382519148143650220 \\
& 1487534725194463844030165897485133626528333829059623064171
\end{aligned}
$$


$e=56109453282671796041582907743795191420565955456458$

70168311956891736518840549653402741454924223492328 55339467393132613402280279316687077146672017196874 20570287613007844191642400792385887828425888389498 23998420028312226377053814767468699311060864836776 42397578959256035850869327112866913028686894236736 38588994494578621026824961704456531411056506148300 25313309529779988125030619797639038338728515848925 16792907351472231296941553085046176147774425005468 73891119031488774848565434843571583946351774298241 76142644520801690300432747947114020655128418504644 3456306876469431878250033702471823625867348033856167261173987

We found $\frac{k}{d}$ at the 306th convergent of $\frac{e}{N^{2}-\frac{9}{4} N+1}$

$k=22303825974699469998859668220910529083895670986870$

31801144199709650127668657563930759368363045074562 2035858758082389737895844930280368787713716478203305 $d=34323988300653048574909503995406966086347176500716$ 52704697231729592771591698828026061279820330727277 488648155695740429018560993999858321906287014145557262923

\subsection{Different bit primes}

In this section, we present our experimental result with Theorem 4 for the case $\mu=6$. We pick two RSA primes $p$ and $q$ such that $q<p<6 q$. The prime $p$ is 513bit and the prime $q$ is 511-bit. The public exponent $e$ is 2025-bit and the secret exponent $d$ is 520 -bit. We can verify that the condition of $d<\frac{N\left(N-\left(\mu+\frac{1}{\mu}\right)\right)}{\sqrt{e\left(\frac{(\mu-1)^{2}}{\mu} N+2\right)}}$ is satisfied as required by Theorem 4 .

$$
\begin{aligned}
p= & 22510842835399690392870027953306607135716421309613 \\
& 16062523970689624877541671394671156390683214685532 \\
& 3666826003631925722143713824982817351298675960251057551 \\
q= & 43094045219164250721440833039123711740424509359250 \\
& 11018528288585049357744490849788167332945916734904 \\
& 453476275039394246312488030728248574860165476490105607
\end{aligned}
$$


$N=97008327907021385390489646654016010166979018555029$ 97030575145759061053369703505120746392756847870272 06659519627031014290641650263276308386538473467834 78941932317363670403674682311276351485982833618775 95377693553973649039487819967937682128431912875915 5743213282914241464625897908161555160091654461709424788457

$e=26716304465047922612125274221514755597537241860791$ 20931327405641923165971868713793092589262478467217 62401622098399694799343177150631461873365667839242 97971366670172935196704721143674735697716376048156 66823014404366414431890369024253618945658356426103 15909863559710607287920009853646203831160061469153 10212966333242652768501539379257580808338675858392 60065080164569283723647842972194446642887134749805 84598396791047808065919102717658994350880770410882 26221621231125991843004472809712982911675436520870 80630515570718647958929769910286907185458180532010 514302732012269579060997422184973437256996239734257519040887

We found $\frac{k}{d}$ at the 291th convergent of $\frac{e}{N^{2}+1-\frac{(\mu+1)^{2}}{2 \mu} N}=\frac{e}{N^{2}+1-\frac{49}{12} N}$

$k=97444221797382625455652208401364783114970194986256$ 17889366405212659933238260849343929429882877863728 92985535727992595886924505233473405159043019272879 $d=34323988300653048574909503995406966086347176500716$ 52704697231729592771591698828026061279820330727277 488648155695740429018560993999858321906287014145557412423

\section{Conclusion}

We have proposed an attack on three variants of the RSA cryptosystem, namely the Kuwakado-Koyama-Tsuruoka extension for singular elliptic curves, Elkamchouchi et al.'s extension of RSA to the Gaussian integer ring and Castagnos scheme. For the three extensions, we showed that the RSA modulus $N=p q$ can be factored in polynomial time if the public exponent $e$ is related to a suitably 
small secret exponent $d$. The attack is based on the theory of continued fractions and can be seen as an extension of Wiener's [12] and Bunder-Tonien's [3] attacks on the RSA.

\section{References}

1. Boneh, D., Durfee, G.: Cryptanalysis of RSA with private key $d$ less than $N^{0.292}$, Eurocrypt'99, Lecture Notes in Computer Science 1592, 1-11, 1999.

2. Bunder, M., Nitaj, A., Susilo, W., Tonien, J.: A new attack on three variants of the RSA cryptosystem, Proceedings of the 21st Australasian Conference on Information Security and Privacy, 2016.

3. Bunder, M., Tonien, J.: A new improved attack on RSA, Proceedings of the 5th International Cryptology and Information Security Conference, 2016.

4. Castagnos, G.: An efficient probabilistic public-key cryptosystem over quadratic field quotients, Finite Fields and Their Applications 13, 563-576, 2007.

5. Coppersmith, D.: Small solutions to polynomial equations, and low exponent RSA vulnerabilities, Journal of Cryptology 10, 233-260, 1997.

6. Elkamchouchi, H., Elshenawy, K., Shaban, H.: Extended RSA cryptosystem and digital signature schemes in the domain of Gaussian integers, Proceedings of the 8th International Conference on Communication Systems, 91-95, 2002.

7. Hardy, G.H., Wright, E.M.: An Introduction to the Theory of Numbers, Oxford University Press, London, 1965.

8. Koyama, K., Maurer, U.M., Okamoto, T., Vanstone, S.A.: New public-key schemes based on elliptic curves over the ring $Z_{n}$, CRYPTO'91, Lecture Notes in Computer Science 576, 252-266, 1991.

9. Kuwakado, H., Koyama, K., Tsuruoka, Y.: A new RSA-type scheme based on singular cubic curves $y^{2}=x^{3}+b x^{2}(\bmod n)$, IEICE Transactions on Fundamentals E78-A,27-33, 1995.

10. Lenstra, H.: Factoring integers with elliptic curves, Annals of Mathematics 126 , 649-673, 1987.

11. Rivest, R., Shamir, A., Adleman, L.: A Method for obtaining digital signatures and public-key cryptosystems, Communications of the ACM 21, 120-126, 1978.

12. Wiener, M.: Cryptanalysis of short RSA secret exponents, IEEE Transactions on Information Theory 36, 553-558, 1990. 